\title{
Proteomic and functional analyses demonstrate the involvement of oxidative stress in the anticancer activities of oridonin in HepG2 cells
}

\author{
HUI WANG ${ }^{1,2}$, YAN YE ${ }^{2}$ and ZHI-LING YU ${ }^{2}$ \\ ${ }^{1}$ Guangdong Provincial Key Laboratory of Biotechnology Candidate Drug Research, School of Life Science and \\ Biopharmaceutics, Guangdong Pharmaceutical University, Guangzhou; ${ }^{2}$ Center for Cancer and Inflammation Research, \\ School of Chinese Medicine, Hong Kong Baptist University, Kowloon Tong, Hong Kong, SAR, P.R. China
}

Received January 3, 2014; Accepted February 27, 2014

DOI: $10.3892 /$ or.2014.3081

\begin{abstract}
Oridonin exhibits a curative effect on liver carcinoma in patients and experimental animals. In the present study, we performed proteomic and functional analyses to explore the mechanism involved in the anticancer activity of oridonin. Oridonin treatment for $24 \mathrm{~h}$ resulted in a dose-dependent decrease in cell viability with an $\mathrm{IC}_{50}$ value of $37.90 \mu \mathrm{M}$. Treatment with $40 \mu \mathrm{M}$ oridonin for $24 \mathrm{~h}$ induced apoptosis as typical apoptotic nuclear alterations were observed following DAPI staining. Using a 2-DE-based proteomic approach, 3 upregulated oxidative stress markers, Hsp70-1, Hop and $\operatorname{Prdx} 2$, were identified in the HepG2 cells treated with $40 \mu \mathrm{M}$ oridonin for $24 \mathrm{~h}$. A pattern of alteration in Hsp70-1 was verified by western blotting. The mRNA expression patterns of Hsp70-1 and Hop as determined by qPCR were comparable to their protein expression patterns. Further investigations showed that oridonin treatment for $24 \mathrm{~h}$ resulted in reactive oxygen species (ROS) generation, and ROS scavenger $\mathrm{N}$-acetylcysteine (NAC) completely inhibited ROS production and restored cell viability, suggesting that oxidative stress contributed to oridonin-induced HepG2 cell death. Western blot analysis of oxidative stress pathway-related proteins demonstrated that oridonin treatment increased p-JNK, p-p38 and p-p53, and decreased Bcl-2 protein expression levels, promoted cytochrome $c$ release, decreased mitochondrial membrane potential, and activated caspase- 9 and caspase- 3 . Furthermore, knockdown of Hsp70-1 expression with specific shRNA significantly decreased the viability of the cells treated with oridonin, suggesting a protective role of Hsp70-1 in oridonin-mediated oxidative stress. The results of the present study provide evidence for a link between oxidative stress and oridonin-induced apoptosis in HepG2 cells.
\end{abstract}

Correspondence to: Dr Zhi-Ling Yu, Center for Cancer and Inflammation Research, School of Chinese Medicine, Hong Kong Baptist University, Kowloon Tong, Hong Kong, SAR, P.R. China E-mail: zlyu@hkbu.edu.hk

Key words: apoptosis, HepG2, oridonin, oxidative stress, proteomics

\section{Introduction}

Rabdosia rubescens (Hemsl.) Hara (Dong Ling Cao in Chinese) is a medicinal herb and has long been used for the treatment of hepatoma in China (1). Oridonin (Fig. 1) is the main active constituent of $R$. Rubescens. Although laboratory and clinical data have confirmed the inhibitory activities of oridonin in hepatocellular carcinoma (2-5), the molecular mechanisms of action of oridonin have not been fully elucidated. In the present study, we found that expression levels of a number of oxidative stress markers were upregulated in oridonin-treated HepG2 cells using a proteomic approach.

Oxidative stress is a disturbance in the oxidant-antioxidant balance leading to potential cellular damage. The imbalance can result from a lack of antioxidant capacity caused by disturbances in production and distribution, or by an overabundance of reactive oxygen species (ROS) from other factors. ROS are potential carcinogens due to their roles in mutagenesis, tumor promotion and progression (6). It is commonly accepted that cancer cells have an increased ROS steady state level and are likely to be more vulnerable to damage by further ROS insults induced by exogenous agents. Thus, manipulating ROS levels by redox modulation could be a way to selectively kill cancer cells without causing significant toxicity to normal cells (7). A unique anticancer strategy termed 'oxidation therapy' has been developed by inducing cytotoxic oxystress for cancer treatment. Many antitumor agents, such as vinblastine, cisplatin, mitomycin $\mathrm{C}$, doxorubicin, camptothecin, inostamycin, neocarzinostatin exhibit antitumor activity via ROS-dependent activation of apoptotic cell death $(8,9)$. To explore the role of oxidative stress in the anticancer activities of oridonin in HepG2 cells, we further performed functional analysis. Results showed that ROS production was responsible for oridonin-induced HepG2 cell death.

\section{Materials and methods}

Reagents. Oridonin was purchased from Shanghai Shamrock Imp. \& Exp. Trading Co., Ltd. (Shanghai, China). Its purity was determined to be $97 \%$ by HPLC. Immobilized $\mathrm{pH}$ gradient (IPG) strips, dithiothreitol (DTT), the 2D Clean-Up 
Kit and the 2D Quant Kit were obtained from GE Healthcare. MTT [3-(4,5-dimethylthiazol-2-yl)-2,5-diphenyltetrazolium bromide] was from USB Corporation. Trypsin was from Promega Corporation (Madison, WI, USA). Silver nitrate, Rhodamine 123 and 4,6-diamidino-2-phenylindole (DAPI) were from Sigma-Aldrich Biotechnology. Power SYBR-Green PCR Master Mixture was obtained from Applied Biosystems. Protease inhibitor cocktail, phosphatase inhibitor cocktail and FuGENE HD transfection reagent were from Roche Bioscience. The mitochondria isolation kit was from Beijing Applygen Technologies Inc. Bio-Rad protein assay was from Bio-Rad Laboratories, Inc. Iodoacetamide (IAA), nitrocellulose membranes and ECL detection reagents were purchased from Amersham Biosciences. p-JNK (Thr183/Tyr185), p-p38 (Thr180/Tyr182), p-p53 (Ser15), cytochrome $c$ and cleaved caspase-3 antibodies were from Cell Signaling Technology. Hsp70, Bcl-2, caspase-3, caspase-9, $\beta$-actin and anti-rabbit IgG antibodies (horseradish peroxidase-conjugated) as well as the Hsp70 shRNA and control shRNA were from Santa Cruz Biotechnology (Santa Cruz, CA, USA). The SuperScript II reverse transcription kit, TRIzol reagent, $\mathrm{CM}-\mathrm{H}_{2}$ DCFDA and the anti-mouse IgG antibody (horseradish peroxidase conjugated) were obtained from Invitrogen Biotechnology.

Cell culture. HepG2 cells (ATCC, Manassas, VA, USA), grown in Dulbecco's modified Eagle's medium (DMEM; Gibco, Carlsbad CA, USA) supplemented with $10 \%$ fetal bovine serum (FBS; Gibco) and $1 \%$ penicillin/streptomycin (P/S, Gibco) were cultured at $37^{\circ} \mathrm{C}$ in an atmosphere containing $5 \%$ $\mathrm{CO}_{2}$.

Determination of cell viability. Cell viability was assessed by the MTT assay. HepG2 cells (5.0x103/well) were seeded and grown in 96-well plates for $24 \mathrm{~h}$ and then treated with various concentrations of oridonin. Control cells were treated with $0.1 \%$ DMSO. After a 24-h incubation, $10 \mu \mathrm{l}$ of $5 \mathrm{mg} / \mathrm{ml}$ MTT solution was added to each well, and the plates were incubated at $37^{\circ} \mathrm{C}$ for $4 \mathrm{~h}$. Following medium removal, $100 \mu \mathrm{l}$ of DMSO was added to each well and the plates were gently shaken for 5 min. Optical absorbance was determined at $570 \mathrm{~nm}$ with a microplate spectrophotometer (BD Biosciences, Franklin Lakes, NJ, USA). The absorbance obtained by vehicle-treated cells was considered to represent $100 \%$ cell survival. Each treatment was performed in triplicate and each experiment was repeated six times.

Fluorescence microscopic analysis of apoptosis. Apoptotic morphological alterations were monitored in DAPI-stained cells. Cells $\left(40 \times 10^{4}\right)$ were grown for $24 \mathrm{~h}$ on coverslips in $35-\mathrm{mm}$ dishes in the presence or absence of $40 \mu \mathrm{M}$ oridonin. Coverslips were carefully washed with PBS, fixed with $4 \%$ paraformaldehyde for $10 \mathrm{~min}$ and incubated with $10 \mu \mathrm{g} / \mathrm{ml}$ DAPI for 10 min. Cells were washed with PBS and observed under a fluorescence microscope (Nikon, Tokyo, Japan).

Proteomic sample preparation and 2-DE. Cells were seeded in $100-\mathrm{mm}$ culture dishes at $1.5 \times 10^{6}$ cells/dish, incubated overnight and then treated with $40 \mu \mathrm{M}$ oridonin for $24 \mathrm{~h}$. Cells were harvested by trypsinization, washed three times with isotonic buffer (10 mM Tris, $250 \mathrm{mM}$ sucrose, $\mathrm{pH}$ 7.2)



Figure 1. Chemical structure of oridonin.

and lysed in a lysis buffer (8 M urea, $4 \%$ CHAPS, $40 \mathrm{mM}$ DTT and 0.5\% IPG buffer pH 3.0-10.0 NL) by gentle shaking for $60 \mathrm{~min}$ on ice. Extracts were centrifuged at $25,000 \mathrm{x} \mathrm{g}$ for $60 \mathrm{~min}$ at $4^{\circ} \mathrm{C}$. Supernatants were purified with the $2 \mathrm{D}$ Clean-Up Kit following the manufacturer's instructions. The protein concentration of each purified sample was determined using the 2D Quant Kit, and protein samples were stored in aliquots at $-80^{\circ} \mathrm{C}$ until further analysis.

First-dimension separation was performed using $13-\mathrm{cm}$ IPG strips (pH 3.0-10.0 NL). Samples were diluted in rehydration solution containing $8 \mathrm{M}$ urea, 2\% CHAPS, $0.5 \%$ IPG buffer, $0.002 \%$ bromophenol blue and $0.28 \%$ DTT to reach a final protein load of $100 \mu \mathrm{g}$ (in $250 \mu \mathrm{l}$ ) per strip. IPG strips were actively rehydrated at $30 \mathrm{~V}$ for $12 \mathrm{~h}$, focused at $500 \mathrm{~V}$ for $2.5 \mathrm{~h}, 1,000 \mathrm{~V}$ for $0.5 \mathrm{~h}$, and then the voltage was increased to $8,000 \mathrm{~V}$ gradually over the next $3 \mathrm{~h}$ and maintained at $8,000 \mathrm{~V}$ for $40,000 \mathrm{Vh}$. Prior to the second-dimension separation, IPG strips were equilibrated in equilibration buffer $(6 \mathrm{M}$ urea, 75 mM Tris- $\mathrm{HCl} \mathrm{pH} \mathrm{8.8,} \mathrm{29.3 \%} \mathrm{glycerol,} \mathrm{2 \%} \mathrm{SDS)} \mathrm{containing}$ $1 \%(\mathrm{w} / \mathrm{v})$ DTT for $15 \mathrm{~min}$ and then in the same equilibration buffer containing $2.5 \%$ IAA for a further $15 \mathrm{~min}$. The seconddimension separation was carried out on $10 \%$ SDS-PAGE (20 mA/gel, $10 \mathrm{~min} ; 25 \mathrm{~mA} / \mathrm{gel}, 260 \mathrm{~min}$ ).

Silver staining and image analysis. Protein spots were visualized by silver staining. Gels were fixed overnight in fixing solution ( $40 \% \mathrm{v} / \mathrm{v}$ methanol and $10 \% \mathrm{v} / \mathrm{v}$ acetic acid) and then treated for 30 min with sensitizing solution (30\% ethanol, $4.05 \% \mathrm{w} / \mathrm{v}$ sodium acetate and $0.2 \%$ sodium thiosulfate). Each gel was washed three times with Milli-Q water for 5 min each, stained for $40 \mathrm{~min}$ in $0.1 \%$ silver nitrate solution and then developed by incubation with a developing solution $(2.5 \% \mathrm{w} / \mathrm{v}$ sodium carbonate and $0.02 \%$ formaldehyde) until protein spots appeared. The developing reaction was terminated by putting gels in the stopping solution $(1.46 \% \mathrm{w} / \mathrm{v}$ EDTA, disodium salt) for $10 \mathrm{~min}$, and finally gels were washed three times with Milli-Q water for 5 min each.

The stained gels were scanned with a LabScan 6.0 software installed image scanner (GE Healthcare, Pittsburgh, PA, USA), and data were analyzed using the ImageMaster 2D Platinum 6.0 software (GE Healthcare). The intensity volume of each spot was processed by background subtraction and total spot volume normalization, and the resulting spot volume percentage was used for comparison. Only those significantly (Student's t-test; $\mathrm{P}<0.05)$ and consistently upregulated or downregulated spots ( $>2$ fold) or spots which appeared or disappeared after treatment in three independent experiments were selected for in-gel digestion and MALDI-TOF-MS/MS analysis. 
In-gel digestion and MALDI-TOF-MS/MS analysis. Protein spots were excised from gels, and each sample was transferred to a 1.5-ml Eppendorf tube and de-stained in a freshly prepared de-staining solution $(15 \mathrm{mM}$ potassium ferricyanate and $50 \mathrm{mM}$ sodium thiosulfate) until the brownish color disappeared. Each de-stained sample was washed in $10 \mathrm{mM}$ ammonium bicarbonate $\left(\mathrm{NH}_{4} \mathrm{HCO}_{3}\right)$ for $5 \mathrm{~min}, 10 \mathrm{mM}$ $\mathrm{NH}_{4} \mathrm{HCO}_{3}$ containing $10 \mathrm{mM}$ DTT for $15 \mathrm{~min}$ at $56^{\circ} \mathrm{C}$, $10 \mathrm{mM} \mathrm{NH}_{4} \mathrm{HCO}_{3}$ containing $55 \mathrm{mM} \mathrm{IAA}$ for $20 \mathrm{~min}$ at room temperature in the dark, and $10 \mathrm{mM} \mathrm{NH}_{4} \mathrm{HCO}_{3}$ containing $50 \% \mathrm{ACN}$ for $15 \mathrm{~min}$. After drying in a Vacufuge concentrator (Eppendorf, Hamburg, Germany), each sample was incubated at $37^{\circ} \mathrm{C}$ overnight in $5 \mu \mathrm{l}$ of $5 \mu \mathrm{g} / \mathrm{ml}$ Trypsin Gold solution. The supernatant was collected and the gel was further extracted with $1 \%$ formic acid. The extracts were combined and dried in a Vacufuge concentrator and resuspended in formic acid for MS analysis. The mass spectra were obtained using the Bruker Autoflex III MALDI-TOF/TOF mass spectrometer (Bruker Daltonics, Billerica, MA, USA). Protein identification was performed automatically by searching the Swiss-Prot 55.3 database using the Mascot 2.04 search engine (Matrix Science, Ltd., London, UK). Database searches were carried out using the following parameters: type of search, MS/MS ion search; enzyme, trypsin; and allowance of one missed cleavage. Carbamidomethylation was selected as a fixed modification and oxidation of methionine was allowed to be variable. The peptide and fragment mass tolerance were set at $50 \mathrm{ppm}$ and $0.5 \mathrm{Da}$, respectively. The instrument was selected as MALDITOF-TOF. Proteins with probability-based MOWSE scores $(\mathrm{P}<0.05)$ were considered to be positively identified.

Western blot analysis. Cells were collected and proteins were extracted with RIPA lysis buffer [50 mM Tris-Cl, 1\% v/v NP-40, $0.35 \%$ w/v sodium deoxycholate, $150 \mathrm{mM} \mathrm{NaCl}, 1 \mathrm{mM}$ EDTA, 1 mM EGTA, pH 7.4, 1 mM phenylmethylsulfonyl fluoride (PMSF), $1 \mathrm{mM} \mathrm{NaF}, 1 \mathrm{mM} \mathrm{Na} \mathrm{VO}_{4}$ ] containing a protease inhibitor cocktail and phosphatase inhibitor cocktail. Mitochondrial and cytoplasmic extracts were prepared with a mitochondria isolation kit following the manufacturer's instructions. The protein concentration was determined using the Bio-Rad protein assay. Individual protein samples (20-50 $\mu \mathrm{g}$ ) were separated by SDS-PAGE and then electrotransferred onto nitrocellulose membranes. The membranes were blocked for 30 min with $3 \%$ skim milk in TBST buffer composed of $50 \mathrm{mM}$ Tris ( $\mathrm{pH} 7.6$ ), $150 \mathrm{mM} \mathrm{NaCl}$ and $0.1 \%$ Tween-20 and incubated with primary antibodies overnight at $4^{\circ} \mathrm{C}$ followed by incubation with horseradish peroxidaseconjugated secondary antibodies. Protein signals were visualized by ECL detection reagents according to the manufacturer's instructions.

$q P C R$. Cells were treated with $40 \mu \mathrm{M}$ oridonin for the indicated durations, and total RNA was isolated using TRIzol reagent according to the manufacturer's protocol. Five micrograms of RNA was used for reverse transcription by oligo-dT using the SuperScript II reverse transcription kit. The qPCR primers were designed as follows: Hsp70-1 (sense 5'-caga acaagcgag ccgtgagg-3' and antisense 5'-tcgtgaatctgggecttg tcc-3'), Hop (sense 5'-tgtaaggaggcggcagacgg-3' and antisense 5'-taaggc gcatggctgggtca-3'). To normalize the amounts of
RNA in samples, a PCR reaction was also performed with primers of $\beta$-actin (sense 5'-gactacctcatgaagatc-3' and antisense 5'-gatccacatctgctggaa-3'). qPCR was performed in a total volume of $20 \mu \mathrm{l}$, with 1X Power SYBR-Green PCR Master Mixture in the 7500 Fast Real-time PCR system (Applied Biosystems, Foster City, CA, USA). All samples were run in triplicate. The cycling parameters for $\mathrm{qPCR}$ reaction included 40 cycles of $95^{\circ} \mathrm{C}$ for $15 \mathrm{sec}$ and $60^{\circ} \mathrm{C}$ for $60 \mathrm{sec}$. The amplification of specific transcripts was confirmed by melting curve profiles generated at the end of the PCR program. Cycle threshold $(\mathrm{Ct})$ values were normalized to $\beta$-actin, and comparative quantification of the target gene was carried out using the $\Delta \Delta \mathrm{CT}$ method.

Transient transfection of Hsp70 shRNA. Transient HSPA1 gene silencing was attained by transfection of Hsp70 shRNA into HepG2 cells using FuGENE HD transfection reagent according to the manufacturer's instruction. HepG2 cells were grown in 6-well plates overnight to achieve $>80 \%$ confluency at the time of transfection and then were transfected with $4 \mu \mathrm{g}$ of Hsp70 shRNA or control shRNA and $6 \mu \mathrm{l} \mathrm{FuGENE}$ HD transfection reagent. The transfection reagents/shRNA complex was diluted with DMEM (FBS and antibiotic-free) to a total transfection volume of $2 \mathrm{ml}$ and incubation was carried out for $6 \mathrm{~h}$ at $37^{\circ} \mathrm{C}$ until the medium was discarded and replaced with growth medium (DMEM with $10 \%$ FBS). Cells were incubated at $37^{\circ} \mathrm{C}$ for a further $30 \mathrm{~h}$ and then subjected to $40 \mu \mathrm{M}$ oridonin treatment for $12 \mathrm{~h}$. The cells were then collected for verification of protein expression by western blotting. Cell viability was determined by MTT assay.

Measurement of intracellular ROS production. The dye, $\mathrm{CM}-\mathrm{H}_{2}$ DCFDA, which becomes fluorescent after cellular oxidation, was used for ROS detection. HepG2 cells were treated with $40 \mu \mathrm{M}$ oridonin in the absence or presence of $2.5 \mathrm{mM}$ NAC, an ROS scavenger. After $24 \mathrm{~h}$, cells were harvested and washed with PBS. The washed cells were resuspended in PBS containing $10 \mu \mathrm{M}$ CM-H ${ }_{2}$ DCFDA and incubated at $37^{\circ} \mathrm{C}$ in the dark for $30 \mathrm{~min}$. The levels of ROS were then determined by flow cytometry.

Observation of mitochondrial membrane potential (MMP). MMP was determined by the fluorescent dye Rhodamine 123. After incubation with $40 \mu \mathrm{M}$ oridonin for $6 \mathrm{~h}$, cells were collected, resuspended in $1 \mu \mathrm{M}$ Rhodamine 123 and stained at $37^{\circ} \mathrm{C}$ for $15 \mathrm{~min}$. The fluorescence intensity of the cells was analyzed by flow cytometry.

Data analysis. All results are expressed as the mean \pm SEM, and statistical analyses were performed using the Student's t-test.

\section{Results}

Oridonin inhibits cell proliferation and induces apoptosis in HepG2 cells. MTT assay showed that treatment with oridonin for $24 \mathrm{~h}$ resulted in a marked decrease in cell viability in a dosedependent manner (Fig. 2A). The $\mathrm{IC}_{50}$ value was determined as $37.90 \mu \mathrm{M}$ for the 24-h treatment from a dose-response curve with GraphPad Prism 5.0 software (GraphPad Software, San 
Table I. Summary of differentially expressed proteins in the oridonin-treated HepG2 cells.

\begin{tabular}{lcccc}
\hline Protein names & MW & pI & MS/MS scores & Fold-change $^{\mathrm{a}}$ \\
\hline Heat shock 70-kDa protein 1 & 70,294 & 5.48 & 212 & +2.08 \\
Hsc70/Hsp90-organizing protein & 63,227 & 6.40 & 88 & $+^{\mathrm{b}}$ \\
Peroxiredoxin-2 & 22,049 & 5.66 & 192 & +2.45 \\
\hline
\end{tabular}

MW, molecular weight; pI, isoelectric point; MS, mass spectrometry; ${ }^{\mathrm{a} F o l d}$-change in the oridonin-treated HepG2 cells at the time-point of $24 \mathrm{~h}$ compared with the control (+, increase; -, decrease). brotein expression was not detected in the control samples.

A

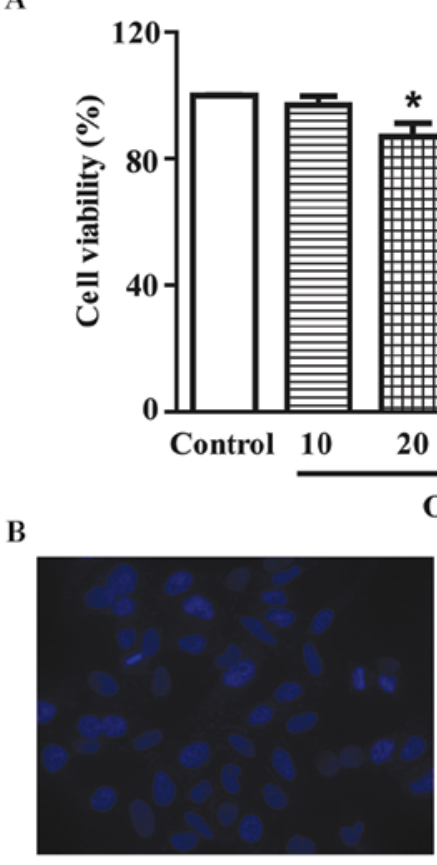

Control

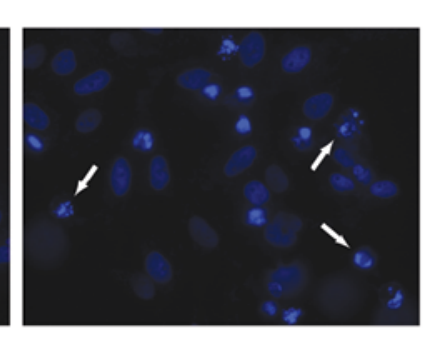

Oridonin
Figure 2. Oridonin inhibits cell growth and induces apoptosis in HepG2 cells. (A) Cells were treated with various concentrations of oridonin or vehicle for 24 h. Cell viability was determined by MTT assay. Data are expressed as the mean \pm SEM from six independent experiments. ${ }^{*} \mathrm{P}<0.05$ vs. the control; ${ }^{* *} \mathrm{P}<0.01$ vs. the control. (B) After a $40 \mu \mathrm{M}$ oridonin treatment for $24 \mathrm{~h}$, the oridonin-treated and control cells were stained with DAPI and visualized by fluorescence microscopy (original magnification, $x 400$ ). Typical apoptotic alterations in nuclei (chromatin condensation, nuclear fragmentation, appearance of apoptotic bodies) are indicated by arrows. Results are representative of three independent experiments.

Diego, CA, USA). In the subsequent assays, $40 \mu \mathrm{M}$ oridonin was used. DAPI staining showed that a 24-h treatment of $40 \mu \mathrm{M}$ oridonin induced the apoptosis of HepG2 cells. Typical apoptotic nuclear alterations (chromatin condensation, nuclear fragmentation, appearance of apoptotic bodies) were observed in the oridonin-treated cells (Fig. 2B).

Differentially expressed proteins in the oridonin-treated HepG2 cells. In an attempt to identify molecular changes following oridonin treatment, we monitored differential protein expression in the HepG 2 cells treated with $40 \mu \mathrm{M}$ oridonin or vehicle for $24 \mathrm{~h}$ using 2-DE-based proteomics. To ascertain reproducibility of results, $2-\mathrm{DE}$ was performed three times for
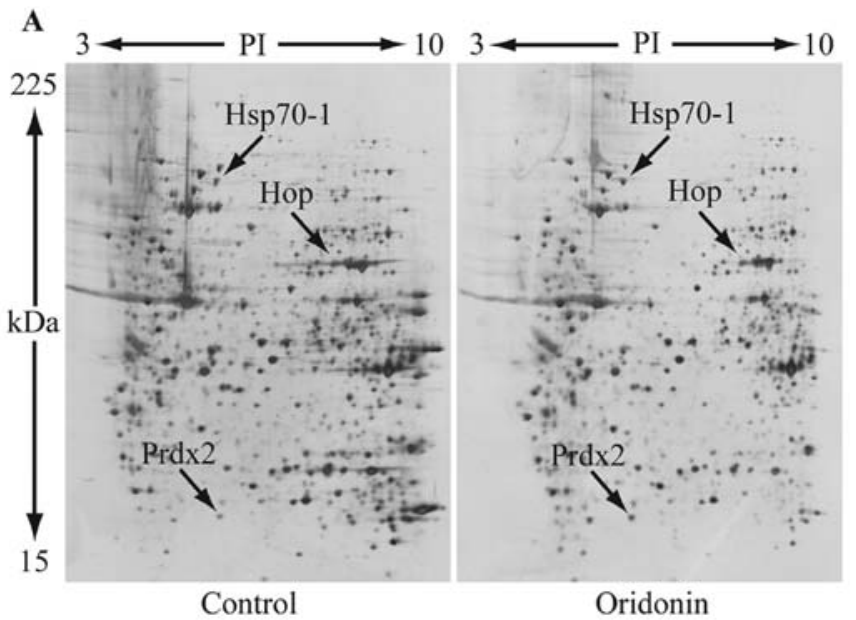

B

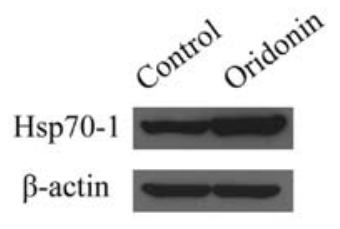

C

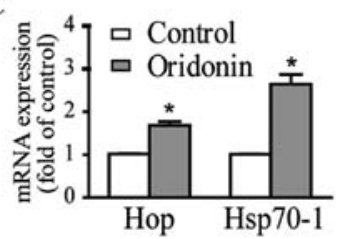

Figure 3. Oridonin treatment is associated with differential expression of Hsp70-1, Hop and Prdx2 in HepG2 cells. Cells were treated with $40 \mu \mathrm{M}$ oridonin or vehicle for $24 \mathrm{~h}$. (A) Representative silver staining images of 2-DE gels with three identified proteins are shown. (B) Western blot analysis of Hsp70-1. $\beta$-actin was included as a protein-loading control. Results are representative of three independent experiments. (C) qPCR analysis of mRNA expression levels of Hsp70-1 and Hop. The comparative Ct method ( $\Delta \Delta \mathrm{Ct}$ method) was used for qPCR data analysis. Data are presented as the mean \pm SEM of three independent experiments. ${ }^{*} \mathrm{P}<0.05$ vs. controls.

each protein sample, and each treatment was repeated three times. Fig. 3A shows the representative gel images. Proteins within the range of $15-225 \mathrm{kDa}$ and with isoelectric point (pI) between 3 to 10 were well separated. Over 1,000 spots were detected on each gel. Three protein spots of interest with fold-changes $>2$ in terms of volume intensity in both triplicate gels and three independent experiments were cut from the gels and analyzed by MALDI-TOF-MS/MS after trypsin digestion. As listed in Table I, these upregulated spots were identified as heat shock 70-kDa protein 1 (Hsp70-1, gene name HSPAl), Hsc70/Hsp90-organizing protein (Hop/Sti1, gene name STIPl) and peroxiredoxin-2 ( $\operatorname{Prdx} 2$, gene name $P R D X 2)$.

The protein expression level of Hsp70-1 in response to $40 \mu \mathrm{M}$ oridonin treatment was verified by western blotting (Fig. 3B). Expression levels of mRNA as determined by qPCR 
A
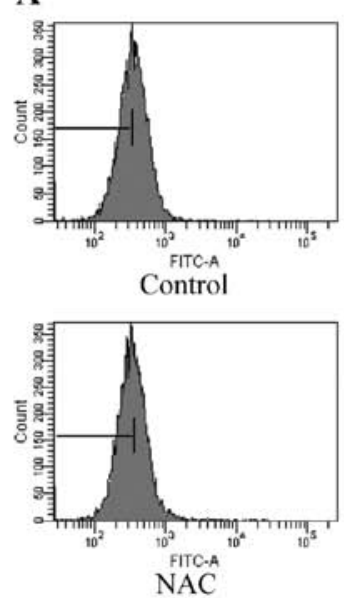

B

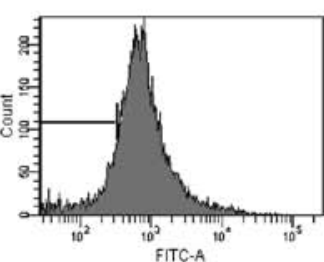

$40 \mu \mathrm{M}$ oridonin

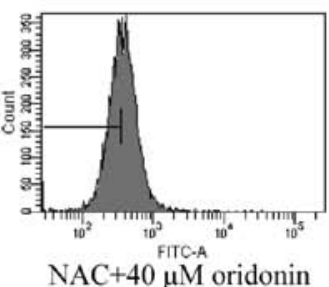

$\mathrm{NAC}+40 \mu \mathrm{M}$ oridonin
C

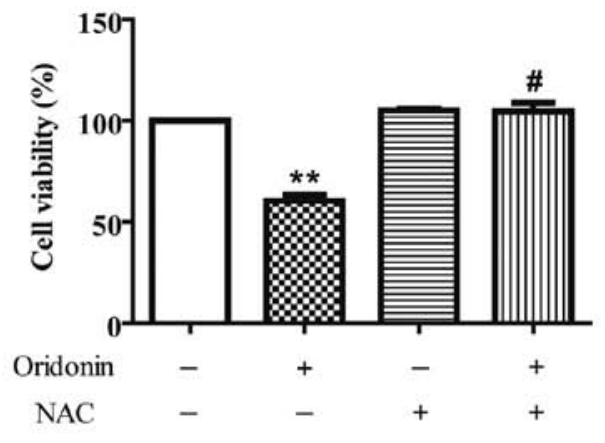

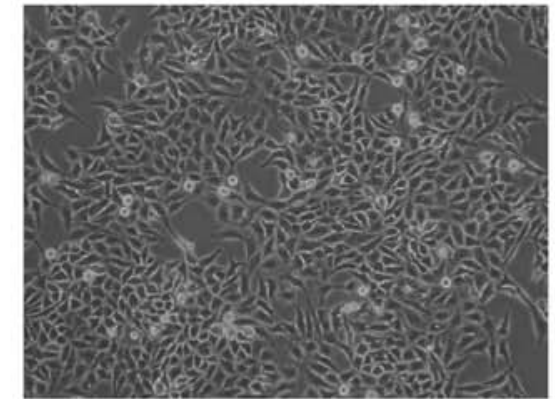

Control

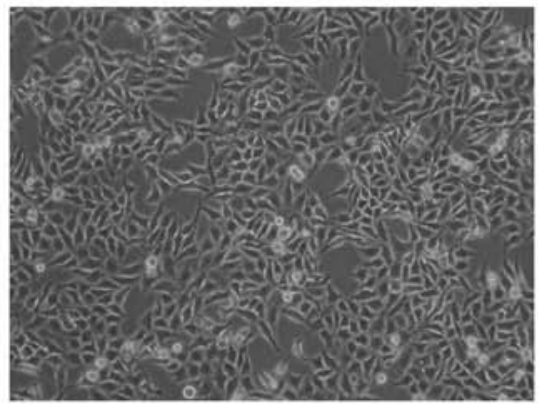

NAC

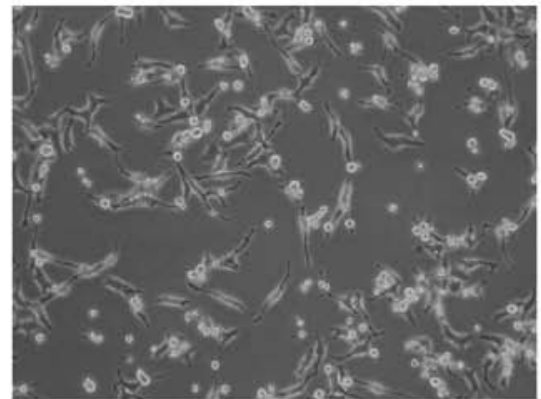

$40 \mu \mathrm{M}$ oridonin

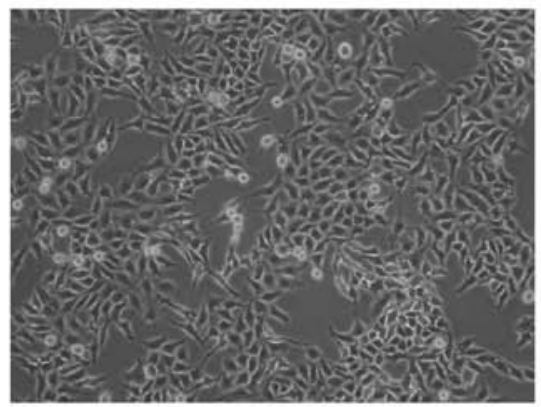

$\mathrm{NAC}+40 \mu \mathrm{M}$ oridonin

Figure 4. Role of ROS in oridonin-induced cell death. (A) Flow cytometric analysis of ROS production. HepG2 cells were stained with ROS-specific probe $\mathrm{CM}-\mathrm{H}_{2}$ DCFDA after the indicated treatments for $24 \mathrm{~h}$. The intracellular fluorescence was determined by flow cytometry. Results are representative of three independent experiments. (B) Representative micrographs of HepG2 cells after the indicated treatments for $24 \mathrm{~h}$. Micrographs were examined by phasecontrast microscopy (original magnification, x100). (C) Effects of NAC on the viability of HepG2 cells treated with $40 \mu \mathrm{M}$ oridonin for 24 h. Cell viability was determined by MTT assay. Data are presented as the mean $\pm \mathrm{SEM}$ of three independent experiments. ${ }^{* *} \mathrm{P}<0.01 \mathrm{vs}$. the control; ${ }^{*} \mathrm{P}<0.01$ vs. $40 \mu \mathrm{M}$ oridonin treatment.

for Hsp70-1 and Hop were 2.64- and 1.68-fold higher than these levels in the control cells (Fig. 3C), which are comparable to their protein expression patterns.

Oridonin-induced ROS production is responsible for cell death in HepG2 cells. Hsp70 acts as a stress sensor and its expression is induced by a variety of stimuli that give rise to cellular oxidation (10). Hop is an extensively studied co-chaperone that not only directly binds to Hsp70 and Hsp90, but also modulates the activities of these chaperones. Overexpression of the Hop gene at the earliest time during stress may be required to mediate the stress response of the inducible Hsp70 (11). Prdx2 belongs to the 2-Cys subgroup of peroxiredoxins (Prdxs).
Prdxs are a family of antioxidative proteins and in mammals the family consists of six members $(12,13)$. They have been reported to be increased in stressed cells induced by oxidants such as $\mathrm{H}_{2} \mathrm{O}_{2}$ (14-16) or ionizing radiation (17). Taken together, the overexpression of Hsp70-1, Hop and Prdx2 indicates that oridonin induces oxidative stress.

To investigate whether oridonin induces oxidative stress in HepG2 cells, the intracellular ROS level was measured by detecting the fluorescent intensity of ROS-specific probe CM- $\mathrm{H}_{2}$ DCFDA after a $40 \mu \mathrm{M}$ oridonin treatment. As shown in Fig. 4A, the ROS level increased at $24 \mathrm{~h}$, and $2.5 \mathrm{mM}$ of the ROS scavenger NAC markedly decreased ROS production in the oridonin-treated HepG2 cells. To explore whether ROS act 
A

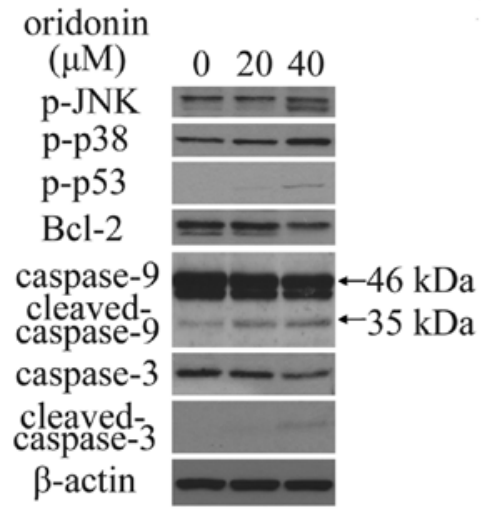

B oridonin
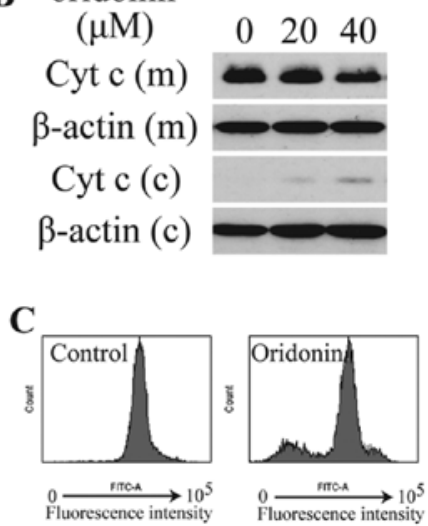

Figure 5. Effects of oridonin on the expression levels of proteins and MMP that are involved in ROS-mediated apoptosis. (A) Dose-dependent effects of oridonin on the expression levels of p-JNK, p-p38, p-p53, Bcl-2, caspase-9, cleaved caspase-9, caspase-3 and cleaved caspase-3. Protein expression in HepG2 cells treated with 0,20 or $40 \mu \mathrm{M}$ oridonin for $24 \mathrm{~h}$ was analyzed by western blotting. $\beta$-actin was included as a protein-loading control. Results shown are representative of three independent experiments. (B) Dose-dependent effects of oridonin on the protein expression levels of cytochrome $c$ (Cyt c). HepG2 cells were treated with 0,20 or $40 \mu \mathrm{M}$ oridonin for $6 \mathrm{~h}$. Mitochondrial (m) and cytoplasmic (c) cytochrome $c$ expression was analyzed by western blotting. $\beta$-actin was included as a protein-loading control. Results shown are representative of three independent experiments. (C) Flow cytometric analysis of mitochondrial membrane potential (MMP). HepG2 cells were treated with or without $40 \mu \mathrm{M}$ oridonin for $6 \mathrm{~h}$ and stained with Rhodamine 123 . Fluorescence intensity of the cells was analyzed by flow cytometry. Results shown are representative of three independent experiments.

as a protective factor in the oridonin-treated HepG2 cells, cells were co-incubated with $2.5 \mathrm{mM}$ NAC and $40 \mu \mathrm{M}$ oridonin for 24 h. NAC completely inhibited oridonin-induced cell death (Fig. 4B). Cell viability was $104.50 \pm 4.31 \%$ in the NAC and oridonin-treated cells vs. $60.49 \pm 2.89 \%$ in the oridonin-treated cells (Fig. 4C). These results suggest that ROS generation plays a role in the antiproliferative activity of oridonin in HepG2 cells.

Oridonin-induced ROS generation results in apoptosis through the activation of JNK and p38 pathways. Oxidative stress resulting from excessive production of ROS triggers apoptotic cell death through p38 and JNK pathways (18). JNK and p38 function as upstream kinases of p53 phosphorylation and subsequently stabilize and activate p53 transcriptional activity, leading to diverse cellular responses, such as apoptosis (19). Bcl-2 and caspase families are required to mediate p53-dependent apoptosis (20).

To determine whether p38 and JNK pathways are involved in the anticancer effects of oridonin in HepG2 cells, immunoblotting was conducted. As shown in Fig. 5A and B, oridonin treatment significantly increased phosphorylated p38 (p-p38), p-JNK and p-p53, and decreased the expression of Bcl-2, mitochondrial cytochrome $c$, caspase-9 (46 kDa) and caspase-3, increased the expression of cytoplasmic cytochrome $c$, as well as activated the cleavage of caspase-9 (35 kDa) and caspase-3. The integrity of the mitochondrial membranes was examined by flow cytometry in Rhodamine 123-stained cells. Results showed that treatment with $40 \mu \mathrm{M}$ oridonin for $6 \mathrm{~h}$ significantly decreased Rhodamine 123 fluorescence intensity (Fig. 5C). This observation suggests that oridonin treatment decreased MMP in HepG2 cells.

Hsp70-1 knockdown increases the antiproliferative effect of oridonin. To investigate the involvement of Hsp70-1 in the apoptotic effect of oridonin, HepG2 cells were transfected with Hsp70 shRNA. Control cells were transfected with the control shRNA plasmid. Oridonin treatment caused Hsp70-1 induction and transient transfection of Hsp70-specific shRNA suppressed Hsp70-1 expression with or without 12-h oridonin treatment (Fig. 6A). Moreover, Hsp70 shRNA transfection, oridonin treatment or the combination of the two treatments significantly reduced cell viability (Fig. 6B and C) when compared with the control cells. These data confirm that Hsp70-1 protects HepG2 cells against cell death. Furthermore, Hsp70-1 knockdown led to the increased sensitivity of HepG2 cells to oridonin cytotoxicity, which suggests that Hsp70-1 acts as a protective factor against oridonin-induced oxidative stress in HepG2 cells.

\section{Discussion}

Oridonin, one of the main bioactive ent-kaurane diterpenoids of $R$. rubescens, possesses anticancer activities. Oridonin can induce G2/M arrest and apoptosis in human hepatocellular carcinoma cells $(21,22)$. Multiple mechanisms such as inhibition of telomerase, regulation of MAPK signaling pathways and induction of endoplasmic reticulum stress have been implicated in the anticancer activity of oridonin $(4,21,23,24)$. In the present study, using a 2-DE-based proteomic approach, we found that the expression levels of oxidative stress markers Prdx2, Hsp70-1 and Hop were altered following oridonin treatment.

Free radicals and reactive molecules containing oxygen such as singlet oxygen, superoxides, peroxides are known as ROS and can cause oxidative stress in cells (18). It has been suggested that oxidative stress in response to a low concentration of ROS may be essential to cellular signaling and proliferation. However, excessive production of ROS may damage various intracellular molecules, leading to severe oxidative stress accompanied by loss of cell function and possibly apoptosis (25). Many studies have shown that apoptosis is closely associated with excessive production of ROS (26-28). Since our proteomic analysis identified several 
A



C

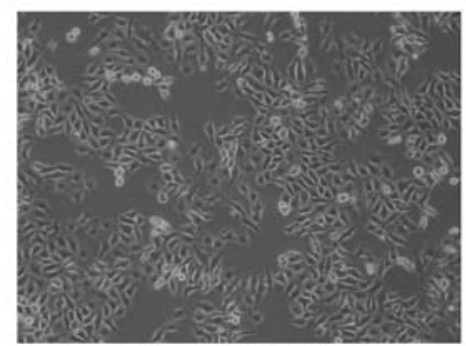

Control shRNA

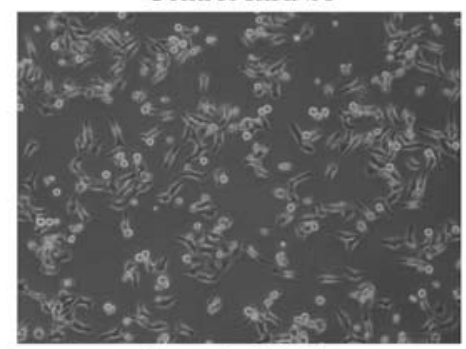

Control shRNA+

$40 \mu \mathrm{M}$ oridonin
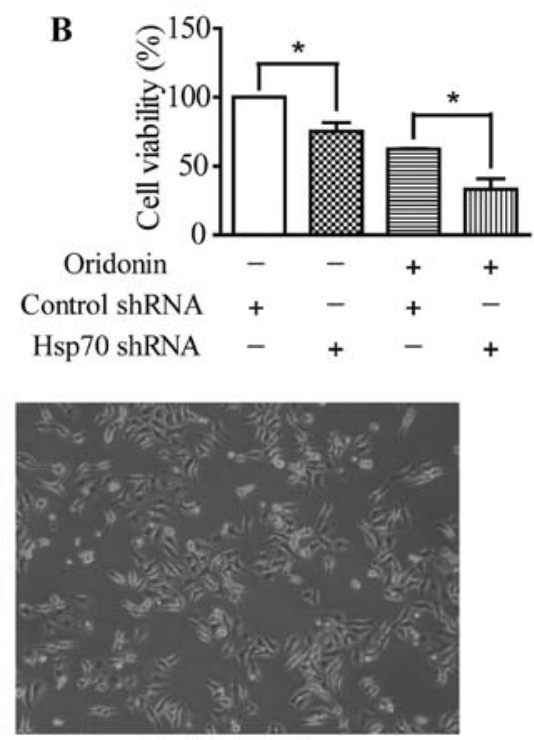

Hsp70 shRNA

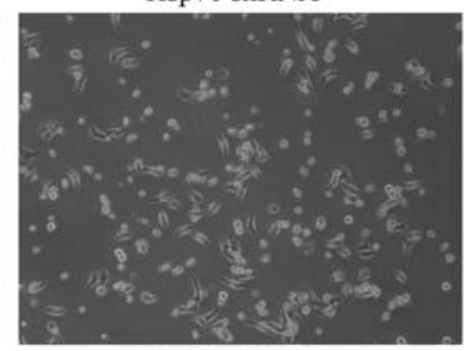

Hsp70 shRNA +

$40 \mu \mathrm{M}$ oridonin

Figure 6. Effects of Hsp70-1 knockdown on cell viability in oridonin-treated HepG2 cells. (A) Western blot analysis of Hsp70-1 expression after Hsp70 shRNA or control shRNA transfection for $48 \mathrm{~h}$. $\beta$-actin was included as a protein-loading control. Results shown were the representative of three independent experiments. (B) Effect of Hsp70-1 knockdown on cell viability in HepG2 cells treated with $40 \mu \mathrm{M}$ oridonin for 12 h. Cell viability was determined by MTT assay. Data were presented as the mean \pm SEM of three independent experiments. ${ }^{*} \mathrm{P}<0.05$. (C) Representative micrographs of HepG 2 cells after indicated treatments for $12 \mathrm{~h}$. Data were obtained under a phase-contrast microscope (original magnification, x100).

proteins related to oxidative stress, we investigated whether oxidative stress occurs in oridonin-treated HepG2 cells and explored the role of oxidative stress in the anticancer activities of oridonin in HepG2 cells. We detected the level of reactive oxygen species (ROS) following oridonin treatment for $24 \mathrm{~h}$ and found that oridonin promoted ROS generation, and ROS scavenger $\mathrm{N}$-acetylcysteine (NAC) completely inhibited ROS production and restored cell viability, suggesting that ROS generation contributed to oridonin-induced HepG2 cell death. Although a low dose of oridonin $(1.4 \mu \mathrm{M})$ is reported to decrease ROS formation and improve cell survival after arsenic treatment (29), therapeutic doses of oridonin are shown to selectively induce apoptosis in various cells through sustained oxidative stress (30-33).

Among many signaling pathways known to be redox sensitive, the MAPK pathway has been well studied. A relative high concentration of ROS may induce activation of JNK and p38 MAPK pathways and contribute to apoptosis $(25,34)$. MAPKs have been shown to phosphorylate p53 at various residues in response to different stressful stimuli, and such phosphorylation can initiate p53 activation and lead to p53-dependent apoptosis (19). Activation of p53 suppresses the expression of anti-apoptotic $\mathrm{Bcl}-2$ protein which prevents the release of mitochondrial cytochrome $c$. Through inhibition of Bcl-2, p53 promotes the release of cytochrome $c$ from the mitochondrial intermembrane space into the cytoplasm (20). Cytochrome $c$ release is frequently coincident with a disruption of the mitochondrial membrane potential (MMP). Released cytochrome $c$ forms an apoptosome complex with caspase-9 and adaptor protein Apaf-1. Caspase-9 is activated following recruitment into the apoptosome and then cleaves and activates effector caspases, such as caspase-3 (35). The cleavage of substrates of caspase-3 significantly alters the cell physiology toward apoptosis (20). We next investigated the involvement of these molecules in oridonin-induced apoptosis by immunoblotting. In oridonin-treated cells, increased expression of p-p38 and p-JNK was accompanied by the upregulation of phosphorylated p53 at serine 5. The activation of p53 decreased the expression of Bcl-2, which triggered mitochondrial cytochrome $c$ release, thus suppressing MMP and activating caspase cascades in HepG2 cells. In agreement with our observations, activation of p38 was reported to be dependent on ROS generation, and p38 and p53 inhibitors have been reported to significantly reduce oridonin-induced apoptosis in HepG2 cells (32).

Abnormal changes in the cellular redox status cause chaperone induction. Chaperones may act as protective factors against cytoplasmic oxidative stress (10). Since upregulation of oxidative stress marker Hsp70-1 was associated with oridonin 
treatment in HepG2 cells, we investigated the role of Hsp70-1 in the antiproliferative effect of oridonin by Hsp70-1 shRNA plasmid transfection. Hsp70-1 knockdown enhanced the sensitivity of HepG2 cells to oridonin-induced cell death. These findings suggest that Hsp70-1 acts as a protective factor against oridonin-induced oxidative stress in $\mathrm{HepG} 2$ cells, and also demonstrates the induction of oxidative stress by oridonin.

Collectively, proteomic analysis revealed that oxidative stress markers Prdx2, Hsp70-1 and Sti1 are involved in the anticancer effects of oridonin. Oxidative stress pathways are activated in oridonin-treated HepG2 cells. Hsp70-1 acts as a protective factor against oxidative stress in $\mathrm{HepG} 2$ cells. These results shed new light on the anti-hepatoma mechanisms of oridonin. Further research is needed to explore the mechanisms by which ROS mediate the apoptotic activity of oridonin.

\section{Acknowledgements}

The present study was supported by a grant (HKBU262512) from the Research Grant Council of Hong Kong and National Natural Science Foundation of China (grant no. 81303292 and 31300737).

\section{References}

1. Gao X, Liu SZ, Liu QL, Liu YY, Zeng DL and Deng MD: Clinical study on anti-cancer effects of Rabdosia rubescens. Chin J Cancer 3: 201-202, 1984.

2. Zhang JF, Chen GH, Lu MQ and Liu JJ: Antiproliferation effects of oridonin on hepatocellular carcinoma BEL-7402 cells and its mechanism. Chin Traditional Patent Med 28: 1325-1329, 2006.

3. Huang J, Wei XY, Sun BH, Wu LJ and Ikejima T: Oridonin induced HepG2 cell death partially through $\mathrm{TNF} \alpha$ signal pathway. Modern Chin Med 12: 28-32, 2010.

4. Cai DT, Jin H, Xiong QX, et al: ER stress and ASK1-JNK activation contribute to oridonin-induced apoptosis and growth inhibition in cultured human hepatoblastoma HuH-6 cells. Mol Cell Biochem 379: 161-169, 2013.

5. Wang RL: Therapeutic effects of Isodon rubescens and oridonin preparations in 31 patients with primary carcinoma of the liver. Ai Zheng 3: 50, 1984.

6. Ha HL, Shin HJ, Feitelson MA and Yu DY: Oxidative stress and antioxidants in hepatic pathogenesis. World J Gastroenterol 16 6035-6043, 2010.

7. Morales-González JA (ed.): Oxidative Stress and Chronic Degenerative Diseases - A Role for Antioxidants. InTech. http:// dx.doi.org/10.5772/45722; doi: 10.5772/2535.

8. Fang J, Nakamura $\mathrm{H}$ and Iyer AK: Tumor-targeted induction of oxystress for cancer therapy. J Drug Target 15: 475-486, 2007.

9. Trachootham D, Alexandre J and Huang P: Targeting cancer cells by ROS-mediated mechanisms: a radical therapeutic approach? Nat Rev Drug Discov 8: 579-591, 2009.

10. Papp E, Nardai G, Soti C and Csermely P: Molecular chaperones, stress proteins and redox homeostasis. Biofactors 17: 249-257, 2003.

11. Odunuga OO, Longshaw VM and Blatch GL: Hop: more than an Hsp70/Hsp90 adaptor protein. Bioessays 26: 1058-1068, 2004.

12. Kang SW, Chae HZ, Seo MS, Kim K, Baines IC and Rhee SG: Mammalian peroxiredoxin isoforms can reduce hydrogen peroxide generated in response to growth factors and tumor necrosis factor- $\alpha$. J Biol Chem 273: 6297-6302, 1998.

13. Kim H, Lee TH, Park ES, et al: Role of peroxiredoxins in regulating intracellular hydrogen peroxide and hydrogen peroxide-induced apoptosis in thyroid cells. J Biol Chem 275: 18266-18270, 2000.

14. Fratelli M, Demol H, Puype M, et al: Identification by redox proteomics of glutathionylated proteins in oxidatively stressed human T lymphocytes. Proc Natl Acad Sci USA 99: 3505-3510, 2002 .
15. Paron I, D'Elia A, D'Ambrosio C, et al: A proteomic approach to identify early molecular targets of oxidative stress in human epithelial lens cells. Biochem J 378: 929-937, 2004.

16. Cesaratto L, Vascotto C, D'Ambrosio C, et al: Overoxidation of peroxiredoxins as an immediate and sensitive marker of oxidative stress in HepG2 cells and its application to the redox effects induced by ischemia/reperfusion in human liver. Free Radic Res 39: 255-268, 2005.

17. Wang T, Tamae D, LeBon T, Shively JE, Yen Y and Li JJ: The role of peroxiredoxin II in radiation-resistant MCF-7 breast cancer cells. Cancer Res 65: 10338-10346, 2005.

18. Pan JS, Hong MZ and Ren JL: Reactive oxygen species: a double-edged sword in oncogenesis. World J Gastroenterol 15: 1702-1707, 2009

19. Wu GS: The functional interactions between the p53 and MAPK signaling pathways. Cancer Biol Ther 3: 156-161, 2004.

20. Shen Y and White E: p53-dependent apoptosis pathways. Adv Cancer Res 82: 55-84, 2001.

21. Wang H, Ye Y, Chui JH, et al: Oridonin induces G2/M cell cycle arrest and apoptosis through MAPK and p53 signaling pathways in HepG2 cells. Oncol Rep 24: 647-651, 2010.

22. Zhang JF, Liu JJ, Liu PQ, Lin DJ, Li XD and Chen GH: Oridonin inhibits cell growth by induction of apoptosis on human hepatocelluar carcinoma BEL-7402 cells. Hepatol Res 35: 104-110, 2006.

23. Wang H, Ye Y, Chu JH, Zhu GY, Fong WF and Yu ZL: Proteomic and functional analyses reveal the potential involvement of endoplasmic reticulum stress and $\alpha-\mathrm{CP} 1$ in the anticancer activities of oridonin in HepG2 cells. Integr Cancer Ther 10: 160-167, 2010.

24. Zhang JF, Chen GH, Lu MQ, Li H, Cai CJ and Yang Y: Change of Bcl-2 expression and telomerase during apoptosis induced by oridonin on human hepatocelluar carcinoma cells. Zhongguo Zhong Yao Za Zhi 31: 1811-1814, 2006 (In Chinese).

25. Yoon SO, Yun $\mathrm{CH}$ and Chung AS: Dose effect of oxidative stress on signal transduction in aging. Mech Ageing Dev 123: 1597-1604, 2002.

26. Liu J, Shen HM and Ong CN: Role of intracellular thiol depletion, mitochondrial dysfunction and reactive oxygen species in Salvia miltiorrhiza-induced apoptosis in human hepatoma HepG2 cells. Life Sci 69: 1833-1850, 2001.

27. Datta K, Babbar P, Srivastava T, Sinha S and Chattopadhyay P: p53 dependent apoptosis in glioma cell lines in response to hydrogen peroxide induced oxidative stress. Int J Biochem Cell Biol 34: 148-157, 2002.

28. Haruna S, Kuroi R, Kajiwara K, et al: Induction of apoptosis in HL-60 cells by photochemically generated hydroxyl radicals. Bioorg Med Chem Lett 12: 675-676, 2002.

29. Du Y, Villeneuve NF, Wang XJ, et al: Oridonin confers protection against arsenic-induced toxicity through activation of the Nrf2-mediated defensive response. Environ Health Perspect 116: 1154-1161, 2008

30. Zang L, He H, Xu Q, et al: Reactive oxygen species $\mathrm{H}_{2} \mathrm{O}_{2}$ and radical $\cdot \mathrm{OH}$, but not $\mathrm{O} 2^{*}$ promote oridonin-induced phagocytosis of apoptotic cells by human histocytic lymphoma U937 cells. Int Immunopharmacol 15: 414-423, 2013.

31. Cheng Y, Qiu F, Ye YC, et al: Autophagy inhibits reactive oxygen species-mediated apoptosis via activating p38-nuclear factor-kappa B survival pathways in oridonin-treated murine fibrosarcoma L929 cells. FEBS J 276: 1291-1306, 2009.

32. Huang J, Wu L, Tashiro S, Onodera S and Ikejima T: Reactive oxygen species mediate oridonin-induced HepG2 apoptosis through p53, MAPK, and mitochondrial signaling pathways. J Pharmacol Sci 107: 370-379, 2008

33. Yu Y, Fan SM, Song JK, Tashiro S, Onodera S and Ikejima T: Hydroxyl radical $(\cdot \mathrm{OH})$ played a pivotal role in oridonin-induced apoptosis and autophagy in human epidermoid carcinoma A431 cells. Biol Pharm Bull 35: 2148-2159, 2012.

34. McCubrey JA, Lahair MM and Franklin RA: Reactive oxygen species-induced activation of the MAP kinase signaling pathways. Antioxid Redox Signal 8: 1775-1789, 2006.

35. Schuler M and Green DR: Mechanisms of p53-dependent apoptosis. Biochem Soc Trans 29: 684-688, 2001. 